\title{
Boundary Super-Deformations, Boundary States and Tachyon Condensation
}

\author{
Zahra Rezaei \\ Physics Department, Tafresh University \\ P.O.Box: 39518-79611, Tafresh, Iran \\ e-mail: z.rezaei@aut.ac.ir
}

\begin{abstract}
The open string tachyon and $U(1)$ gauge field as longitudinal fluctuations and the velocity as transverse fluctuation of an arbitrary dimensional $D$-brane are considered as boundary deformations of a closed superstring free action. The path integral approach will be applied to calculate the corresponding generalized boundary states using supersymmetrized boundary actions. Obtaining the disk partition functions from the boundary states and studying the effect of tachyon condensation on both of them in the NSNS and RR sectors, leads to results that differ from the established ones.
\end{abstract}

PACS numbers: 11.25.-w; 11.25.Hf

Keywords: Superstring, Boundary state, $D p$-brane, Partition function, Tachyon condensation 


\section{Introduction}

$D$-branes, as unavoidable objects of string theory, can be studied through two different approaches. On one hand, we can regard $D$-branes as open strings boundaries because open strings are quantum excitations of $D$-branes [1,2]. On the other hand, since the boundary itself shows the creation out of vacuum [3], we can provide any $D$-brane with a boundary state that represents the closed string creation and shows the coupling of all closed string states to the $D$-brane [4].

The $U(1)$ gauge field (photon) and tachyon are two important states in open string spectrum so that the former appears because of the ending of open strings on the $D$-brane, and the latter points out the instability of the $D$-brane. These are in fact fluctuations along the $D$-brane world volume while the $D$-brane itself as a dynamic object can be influenced by transverse fluctuations, too. These transverse fluctuations, which are equivalent to taking into account scalar fields from the world sheet point of view [5], can be interpreted as $D$ brane velocity. Boundary states corresponding to each of these deformations (longitudinal and transverse) have been investigated, separately in different papers [3, 66 17]. But our main task in this article is taking into account these longitudinal and transverse fluctuations simultaneously as supersymmetrized deformations of the original theory and obtaining more generalized boundary states by the path integral method.

Actually open strings ending on bosonic and non-BPS $D$-branes, and also stretched between $D \bar{D}$-branes contain tachyon that makes these systems unstable. Because of the tachyon influence, an unstable $D$-brane decays to lower dimensional configurations, and this

process is called tachyon condensation [18 21]. During this process the negative energy density of the tachyon potential at its minimum point, cancels the tension of the $D$-brane (or $D$-branes) [22], and the final product is a closed string vacuum without a $D$-brane or stable lower dimensional $D$-branes [23, 24].

Studying tachyon condensation is possible via two main tools, open string field theory [2527] and boundary string field theory [18,28 33. The discussion about tachyon condensation using boundary state formalism, which is our approach in this article, is closely related to the latter because the boundary state normalization factor corresponds to disk partition function which is the main component of the latter approach.

In our previous paper [11] we have also considered at the same time the presence of the $U(1)$ gauge field, the tachyon field, and the velocity of the $D p$-brane, but the procedure of calculating the boundary state was completely different. Besides, in [1] the main goal was calculating the cylindrical amplitude between two $D p_{1}-D p_{2}$-branes while in this article we 
are interested in disk partition functions and the effect of tachyon condensation on them.

So in this article we consider a $U(1)$ gauge field and tachyon both living on a $D p$ brane world volume with arbitrary dimension. Then, we let the $D p$-brane have velocity along normal directions to its world volume. Each one of these longitudinal and transverse fluctuations will be added as a boundary action to the free action of the theory. Then, we will introduce superfields, bosonic and fermionic boundary coordinates, and boundary superderivatives to find the supersymmetrized form of the mentioned deformations, which is one of the goals of this article. Having boundary actions, bosonic and fermionic boundary states are calculated by the path integral approach. The profound relation between the boundary state and the disk partition function will help us to find the NSNS and RR partition functions. Finally, the effect of the tachyon and its condensation on boundary states will be investigated.

Simultaneous consideration of longitudinal and transverse fluctuations (in spite of some technical difficulties), studied in the framework of superstring theory and taking into account zero modes of boundary actions and their role in the boundary state, are the main distinctions from the conventional literature. This generality has caused interesting deviations from standard results, both in the boundary state and the tachyon condensation discussion, to appear. Briefly, the disk partition function (as the normalization factor of the boundary state) lacks the conventional dependence on the tachyon and it causes the process of tachyon condensation to be different. In fact, during tachyon condensation the dimensional reduction of the $D p$-brane occurs but the tachyon does not completely vanish and affects the boundary state of the newly constructed $D$-brane in the form of a constant factor.

\section{Bulk action and basis boundary states}

In order to calculate the full boundary state of a moving $D p$-brane in the presence of a background tachyon and $\mathrm{U}(1)$ gauge field, the full sigma-model action of the closed superstring is needed. This action can be divided into two parts, bulk and boundary. The boundary actions will be investigated comprehensively in the next section. Bulk action actually is the superstring free action in $\mathrm{d}=10$ dimensional spacetime, and its form in terms of superfield $Y^{\mu}$ is

$$
S=-\frac{1}{4 \pi \alpha^{\prime}} \int_{\Sigma} d z d \bar{z} d \vartheta d \bar{\vartheta} g_{\mu \nu} D Y^{\mu} \bar{D} Y^{\nu}
$$

where

$$
z=\sigma+i \tau, \quad \bar{z}=\sigma-i \tau
$$


and

$$
\vartheta=\vartheta_{+}+i \vartheta_{-}, \quad \bar{\vartheta}=\vartheta_{+}-i \vartheta_{-}
$$

$(\sigma, \tau)$ and $\left(\vartheta_{+}, \vartheta_{-}\right)$are bosonic and Grassmann coordinates of the world sheet $\Sigma$, respectively [34]. $D$ and $\bar{D}$ are superderivatives that can be shown as

$$
\left\{\begin{array}{l}
D=i \frac{\partial}{\partial \vartheta}+\frac{1}{2} \bar{\vartheta} \frac{\partial}{\partial z} \\
\bar{D}=i \frac{\partial}{\partial \vartheta}+\frac{1}{2} \vartheta \frac{\partial}{\partial \bar{z}}
\end{array}\right.
$$

The superfield $Y^{\mu}$ is defined in terms of spacetime coordinates $X^{\mu}$ and their fermionic partners $\psi^{\mu}$

$$
Y^{\mu}=X^{\mu}+\vartheta \psi_{+}^{\mu}+i \eta \bar{\vartheta} \psi_{-}^{\mu}+i \vartheta \bar{\vartheta} B^{\mu}
$$

where $\psi_{+}^{\mu}$ and $\psi_{-}^{\mu}$ are the components of the doublet $\psi^{\mu}$

$$
\psi^{\mu}=\left(\begin{array}{c}
\psi_{+}^{\mu} \\
\psi_{-}^{\mu}
\end{array}\right)
$$

Combining all the above relations, superstring action (1) can be expressed in the RNS formulation as follows:

$$
S=-\frac{1}{4 \pi \alpha^{\prime}} \int_{\Sigma} d^{2} \sigma\left(g_{\mu \nu} \partial_{a} X^{\mu} \partial^{a} X^{\nu}-i g_{\mu \nu} \bar{\psi}^{\mu} \rho^{a} \partial_{a} \psi^{\nu}\right)
$$

where $\rho$ 's are Dirac matrices in two dimensions and $g_{\mu \nu}$ is constant. $X^{\mu}$ and $\psi^{\mu}$ as the solution of closed superstring equations of motion are defined in terms of their oscillating and zero modes as

$$
\begin{aligned}
& X^{\mu}(\sigma, \tau)= x_{0}^{\mu}+2 \alpha^{\prime} p^{\mu} \tau+\sqrt{\frac{\alpha^{\prime}}{2}} \sum_{m>0} m^{-1 / 2}\left(x_{m}^{\mu} e^{2 i m \sigma}+\bar{x}_{m}^{\mu} e^{-2 i m \sigma}\right), \\
& \psi_{+}^{\mu}=\sum_{m>0}\left\{\tilde{\psi}_{m}^{\mu} e^{-2 i m(\tau+\sigma)}+\tilde{\psi}_{-m}^{\mu} e^{2 i m(\tau+\sigma)}\right\} \\
& \psi_{-}^{\mu}=\sum_{m>0}\left\{\psi_{m}^{\mu} e^{-2 i m(\tau-\sigma)}+\psi_{-m}^{\mu} e^{2 i m(\tau-\sigma)}\right\}
\end{aligned}
$$

In the expansion (4) $p^{\mu}$ is the closed superstring momentum and, $x$ and $\bar{x}$ are linear combinations of the bosonic oscillators $a$ and $\tilde{a}$

$$
\left\{\begin{array}{l}
x_{m}=a_{m} e^{-2 i m \tau}+\tilde{a}_{m}^{\dagger} e^{2 i m \tau} \\
\bar{x}_{m}=a_{m}^{\dagger} e^{2 i m \tau}+\tilde{a}_{m} e^{-2 i m \tau}
\end{array}\right.
$$

where the standard harmonic oscillators $a(\tilde{a})$ and $a^{\dagger}\left(\tilde{a}^{\dagger}\right)$ are related to annihilation and creation operators $\alpha(\tilde{\alpha})$ and $\alpha^{\dagger}\left(\tilde{\alpha}^{\dagger}\right)$ in the following manner

$$
a_{m}^{\mu}=\frac{i}{\sqrt{m}} \alpha_{m}^{\mu}, \quad a_{m}^{\dagger \mu}=\frac{-i}{\sqrt{m}} \alpha_{-m}^{\mu}, \quad \tilde{a}_{m}^{\mu}=\frac{i}{\sqrt{m}} \tilde{\alpha}_{m}^{\mu}, \quad \tilde{a}_{m}^{\dagger \mu}=\frac{-i}{\sqrt{m}} \tilde{\alpha}_{-m}^{\mu} .
$$


Also $\tilde{\psi}_{m}^{\mu}\left(\tilde{\psi}_{-m}^{\mu}\right)$ and $\psi_{m}^{\mu}\left(\psi_{-m}^{\mu}\right)$ are fermionic oscillators in (5) and (6). Defining $\theta^{\mu}$ as the boundary fermion, it should be written as a linear combination of $\psi_{+}^{\mu}$ and $\psi_{-}^{\mu}$

$$
\theta^{\mu}=\psi_{+}^{\mu}+i \eta \psi_{-}^{\mu}
$$

By considering the following oscillating form for $\theta^{\mu}$

$$
\theta^{\mu}=\sum_{m>0}\left(\theta_{m}^{\mu} e^{-2 i m \sigma}+\bar{\theta}_{m}^{\mu} e^{2 i m \sigma}\right)
$$

its components $\theta_{m}^{\mu}$ and $\bar{\theta}_{m}^{\mu}$ are defined as a combination of fermionic oscillators

$$
\left\{\begin{aligned}
\bar{\theta}_{m}^{\mu} & =\tilde{\psi}_{m}^{\mu \dagger} e^{2 i m \tau}+i \eta \psi_{m}^{\mu} e^{-2 i m \tau} \\
\theta_{m}^{\mu} & =\tilde{\psi}_{m}^{\mu} e^{-2 i m \tau}-i \eta \psi_{m}^{\mu \dagger} e^{2 i m \tau}
\end{aligned}\right.
$$

in analogy with (7) for the bosonic part. The relations between fermionic oscillators are

$$
\tilde{\psi}_{-m}^{\mu}=\tilde{\psi}_{m}^{\mu \dagger} \quad, \quad \psi_{-m}^{\mu}=-\psi_{m}^{\mu \dagger} .
$$

Equations (7) and (10) can be considered as the eigenvalue equations [3], and the corresponding eigenstates for $\tau=0$ are

$$
|x, \bar{x}\rangle=\prod_{m=1}^{\infty} \exp \left(-\frac{1}{2} \bar{x}_{m} x_{m}-a_{m}^{\dagger} \tilde{a}_{m}^{\dagger}+a_{m}^{\dagger} x_{m}+\bar{x}_{m} \tilde{a}_{m}^{\dagger}\right)|v a c\rangle,
$$

as the bosonic state and

$$
|\theta, \bar{\theta}\rangle=\prod_{m=1}^{\infty} \exp \left(-\frac{1}{2} \bar{\theta}_{m} \theta_{m}+i \eta \psi_{m}^{\dagger} \tilde{\psi}_{m}^{\dagger}+\psi_{m}^{\dagger} \theta_{m}-i \eta \bar{\theta}_{m} \tilde{\psi}_{m}^{\dagger}\right)|v a c\rangle
$$

as the fermionic state. Actually, these states are basis boundary states resulting from the action (3) which is not accompanied by any deformations. These sets of basis boundary states can be used to make more complicated boundary states related to nontrivial backgrounds that couple to the original theory.

Before introducing boundary actions coupled to the original theory we need to determine the world sheet boundary. Here we set the boundary of the closed string world sheet at $\tau=0$ and $\vartheta_{-}=0$ so the coordinates of this boundary are $\left(\sigma, \vartheta_{+}\right)$. Besides, tangential and normal boundary derivatives are

$$
\left\{\begin{array}{c}
D+\left.\bar{D}\right|_{\left(\tau=0, \vartheta_{-}=0\right)} \equiv D_{t}=i \partial_{\vartheta_{+}}+\frac{1}{2} \vartheta_{+} \partial_{\sigma} \\
D-\left.\bar{D}\right|_{\left(\tau=0, \vartheta_{-}=0\right)} \equiv D_{n}=-\partial_{\vartheta_{-}}-\frac{i}{2} \vartheta_{+} \partial_{\tau}
\end{array}\right.
$$

which we need in the next section to couple the boundary deformations to the theory. 


\section{Boundary actions}

After writing the supersymmetrized free action, (3), to have generalized boundary states we should add boundary actions corresponding to boundary deformations. These boundary actions will be supersymmetrized by construction. As mentioned before, these deformations are open string tachyon, $U(1)$ gauge field and the velocity of the $D p$-brane so that the former fields are parallel to the $D p$-brane while the latter is normal to it. We show the directions along the $D p$-brane with $X^{\alpha}$ where $\alpha \in\{0,1, \ldots, p\}$ and the directions perpendicular to the $D p$-brane with $X^{i}$ so that $i \in\{p+1, \ldots, d-1\} . p$ and $d$ respectively are the $D p$-brane and spacetime dimensions. Also, hereafter we put $\alpha^{\prime}=1$ for further convenience where we want to compare bosonic and fermionic partition functions.

\subsection{Photon}

Photons are massless particles and therefore an important part of the open string spectrum. Since deformations related to open string states couple to the original theory via boundary terms, the bosonic case vector potential $A^{\mu}$ (photon) of the gauge field $U(1)$ couples to the closed string world sheet such as $S_{F} \sim \int_{\partial \Sigma} d \sigma F_{\alpha \beta} X^{\alpha} \partial_{\sigma} X^{\beta}$. In this action $F_{\alpha \beta}$ is the field strength of $A^{\mu}$ and $\partial_{\sigma}$ is derivative along the boundary. Also, $\partial \Sigma$ shows the boundary of the world sheet. Since the $U(1)$ gauge field originates from the ending of the open string on the $D p$-brane, so $F$ is an antisymmetric $(p+1) \times(p+1)$ matrix with components along the $X^{\alpha}$ directions.

In analogy with the above bosonic $S_{F}$, by substituting superfield $Y^{\mu}$ instead of $X^{\mu}$ and tangential superderivative $D_{t}$ instead of $\partial_{\sigma}$, we can write the supersymmetric form of $S_{F}$ as

$$
S_{F}=\frac{1}{2 \pi} \int_{\partial \Sigma} d \sigma d \vartheta_{+} F_{\alpha \beta} Y^{\alpha} D_{t} Y^{\beta}
$$

Now we make use of the complete form of superfield $Y^{\mu},(2)$, and perform Grassmannian integration over $\vartheta_{+}$to find the explicit supersymmetric form of $S_{F}$ as the follows:

$$
S_{\mathrm{F}}=\frac{1}{2 \pi} \int_{\partial \Sigma} d \sigma F_{\alpha \beta}\left(X^{\alpha} \partial_{\sigma} X^{\beta}+i \theta^{\alpha} \theta^{\beta}\right)
$$

where $\theta$ is the boundary fermion. Expansions of $X$ and $\theta$ in terms of their oscillators help us to write the following bosonic and fermionic forms of photon boundary action:

$$
\begin{gathered}
S_{F}^{b}=i \sum_{m>0} F_{\alpha \beta} \bar{x}_{m}^{\alpha} x_{m}^{\beta}, \\
S_{F}^{f}=i \sum_{r>0} F_{\alpha \beta} \bar{\theta}_{r}^{\alpha} \theta_{r}^{\beta} .
\end{gathered}
$$


Upper indices $b$ and $f$ in (15) and (16) stand for bosonic and fermionic, respectively. The index $F$ indicates that the boundary action is related to the $U(1)$ gauge field. The mode number $m$ in the bosonic part runs over the integers while the mode number $r$ in the fermionic part runs over the integers in the $\mathrm{R}$ sector and half-integers in the NS sector. In the bosonic part there is no contribution of zero modes but since $r$ chooses integers in the $\mathrm{R}$ sector of the fermionic part, there is a zero mode contribution in the boundary action from this sector as

$$
S_{F}^{0}=i F_{\alpha \beta} \bar{\theta}_{0}^{\alpha} \theta_{0}^{\beta}
$$

\subsection{Velocity}

To obtain the boundary state corresponding to a moving $D p$-brane, the boundary state in the presence of a stationary $D p$-brane can be obtained and then be affected by the boost operator [7]. However, there is another equivalent method [13] in which the $D p$-brane velocity is considered as transverse fluctuations to the $D p$-brane and so can be added as a boundary term to the original action of the theory. Accordingly, the boundary action due to the $D p$ brane velocity in the bosonic case is $S_{V} \sim \int_{\partial \Sigma} d \sigma X^{0} V^{i} \partial_{\tau} X^{i}$, with $V^{i}$ the $D p$-brane velocity along the $X^{i}$ direction and $\partial_{\tau}$ the normal derivative to the boundary. Consequently its supersymmetric form can be written by analogy as

$$
S_{V}=\frac{1}{2 \pi} \int_{\partial \Sigma} d \sigma d \vartheta_{+} Y^{0} V^{i} D_{n} Y^{i}
$$

where $D_{n}$ is normal superderivative to the boundary. When we use the complete form of $Y^{\mu},(2)$, and $D_{n},(13)$, the supersymmetric $S_{V}$ is given by

$$
S_{V}=\frac{1}{2 \pi} \int_{\partial \Sigma} d \sigma V^{i}\left(X^{0} \partial_{\tau} X^{i}-i\left(\psi_{+}^{0}+i \eta \psi_{-}^{0}\right)\left(\psi_{+}^{i}-i \eta \psi_{-}^{i}\right)\right) .
$$

Careful calculation gives the velocity action in terms of oscillating modes in the bosonic and fermionic sectors as the following forms:

$$
\begin{gathered}
S_{V}^{b}=\frac{i}{2} V^{i} \sum_{m>0}\left\{\bar{x}_{m}^{0}\left(\tilde{a}_{m}^{i \dagger}-a_{m}^{i}\right)+\left(a_{m}^{i \dagger}-\tilde{a}_{m}^{i}\right) x_{m}^{0}\right\}, \\
S_{V}^{f}=-\frac{i}{2} V^{i} \sum_{r>0}\left\{\bar{\theta}_{r}^{0}\left(\tilde{\psi}_{r}^{i}+i \eta \psi_{r}^{i \dagger}\right)-\left(\tilde{\psi}_{r}^{i \dagger}-i \eta \psi_{r}^{i}\right) \theta_{r}^{0}\right\} .
\end{gathered}
$$

Contribution of zero modes in the velocity action limits to the bosonic part because the equality of $\theta_{0}^{0}=\bar{\theta}_{0}^{0}$ causes zero mode terms in the $\mathrm{R}$ sector of the fermionic part to cancel each other, so

$$
S_{V}^{0}=V^{i} x_{0}^{0} p^{i}
$$

according to (4). 


\subsection{Tachyon}

Tachyon is an inevitable part of the bosonic open and closed string spectrum. Although in superstring theories closed string tachyons are removed by GSO projection, there are still combinations in these theories that include tachyon. What we want to do here is consider the open string tachyon as a deformation to original theory that appears as a coupling to the boundary of the closed string world sheet. This coupling in the bosonic case is $S_{T} \sim \int_{\partial \Sigma} d \sigma T(X)$, where $T(X)$ is the tachyon profile. In superstring theory we introduce

$$
\Gamma^{\mu}=x^{\mu}+\vartheta \chi_{+}^{\mu}+i \eta \bar{\vartheta} \chi_{-}^{\mu}+i \vartheta \bar{\vartheta} B^{\mu},
$$

as an auxiliary superfield in which $x^{\mu}$ and $\chi^{\mu}$ are analogous to $X^{\mu}$ and $\psi^{\mu}$ in the main superfield $Y^{\mu}$. By considering $T(Y)$ as a function of superfield $Y^{\mu}$, the corresponding action can be written in the following form:

$$
S_{\mathrm{T}}=\frac{1}{2} \int d \sigma d \vartheta_{+}\left(\Gamma D_{t} \Gamma+T(Y) \Gamma\right),
$$

so that $D_{t}$ is the tangential derivative to the boundary. After expanding $T(Y)$, using the $\Gamma$ and $D_{t}$ relations, (22) and (13), and applying the Grassmannian integration over $\vartheta_{+}, S_{T}$ is obtained as

$$
S_{\mathrm{T}}=\frac{1}{2} \int_{\partial \Sigma} d \sigma\left(i T^{2}+\left(\theta^{\mu} \partial_{\mu} T\right) \partial_{\sigma}^{-1}\left(\theta^{\nu} \partial_{\nu} T\right)\right) .
$$

Since the components of the auxiliary field do not appear in the bulk action, they can completely be eliminated by their equations of motion. This fact has been applied to obtain (23). To have a Gaussian integral we consider a linear profile for the tachyon, i.e. $T^{2}(X)=$ $X^{\mu} u_{\mu \nu} X^{\nu}$, so that $u_{\mu \nu}$ is a constant symmetric matrix. $\partial_{\sigma}^{-1}$ actually is a Green function that by using its following form in terms of the sign function $\epsilon(x)$,

$$
\partial_{\sigma}^{-1} f(\sigma)=\frac{1}{2} \int d \sigma^{\prime} \epsilon\left(\sigma-\sigma^{\prime}\right) f\left(\sigma^{\prime}\right) \quad, \quad \epsilon(x)= \begin{cases}-1 & x<0 \\ 1 & x>0\end{cases}
$$

the bosonic and fermionic parts of the tachyon action are derived in terms of oscillators as

$$
\begin{gathered}
S_{T}^{b}=i \sum_{m>0} \frac{\pi u_{\alpha \beta}}{2 m} \bar{x}_{m}^{\alpha} x_{m}^{\beta}, \\
S_{T}^{f}=i \sum_{r>0} \frac{\pi u_{\alpha \beta}}{2 r} \bar{\theta}_{r}^{\alpha} \theta_{r}^{\beta} .
\end{gathered}
$$

Because the tachyon lives on the $D p$-brane world volume, $u$ is a $(p+1) \times(p+1)$ matrix that has components along the $X^{\alpha}$ directions. 
Furthermore, just the bosonic part contributes in the zero mode action as

$$
S_{T}^{0}=\frac{i \pi}{2} u_{\alpha \beta} \bar{x}_{0}^{\alpha} x_{0}^{\beta}
$$

It seems that there is a contribution of zero modes in the $\mathrm{R}$ sector in which $r$ is an integer. But careful calculation of (23) for $r=r^{\prime}=0$ shows that $\int_{0}^{\pi} d \sigma\left(\theta_{0}+\bar{\theta}_{0}\right) \frac{1}{2} \int_{0}^{\pi} d \sigma^{\prime} \epsilon\left(\sigma-\sigma^{\prime}\right)\left(\theta_{0}+\bar{\theta}_{0}\right)$ is equal to zero. So the fermionic part has no role in the tachyon zero mode action.

\section{Boundary States and disk partition functions}

For an arbitrary boundary action $S_{\text {boundary, bosonic and fermionic boundary states are defined }}$ as

$$
\begin{aligned}
& \left|B ; S_{\text {boundary }}^{b}\right\rangle^{\text {bosonic }}=\int D[x, \bar{x}] e^{i S_{\text {boundary }}^{b}}|x, \bar{x}\rangle, \\
& \left|B ; S_{\text {boundary }}^{f}\right\rangle^{\text {fermionic }}=\int D[\theta, \bar{\theta}] e^{i S_{\text {boundary }}^{f}}|\theta, \bar{\theta}\rangle,
\end{aligned}
$$

where $|x, \bar{x}\rangle$ and $|\theta, \bar{\theta}\rangle$ are basis bosonic, (11), and fermionic, (12), boundary states, respectively. Boundary states (27) and (28) are due to inclusion of external background fields which present in the form of boundary terms added to the original action. These boundary terms are called deformations because they disturb the CFT properties of the world sheet. $D[x, \bar{x}]$ and $D[\theta, \bar{\theta}]$ show the path integral over $x, \bar{x}, \theta$, and $\bar{\theta}$.

To write the total boundary action we should add the boundary actions corresponding to the boundary deformations, (15)-(17), (19)-(21) and (24)-(26). Moreover, the bulk action itself contributes to the boundary. The oscillating part of this contribution helped us to form basis boundary states $|x, \bar{x}\rangle$ and $|\theta, \bar{\theta}\rangle$, and the zero mode part is included in the following boundary actions:

$$
\begin{aligned}
S_{\text {boundary }}^{b}=S_{F, T, V}^{b}=\quad & V x_{0}^{0} p^{i_{0}}+\frac{i \pi}{2} u_{\alpha \beta} \bar{x}_{0}^{\alpha} x_{0}^{\beta}-\frac{1}{2} g_{\mu \nu} x_{0}^{\mu} p^{\nu} \\
& +i \sum_{m>0} F_{\alpha \beta} \bar{x}_{m}^{\alpha} x_{m}^{\beta}+i \sum_{m>0} \frac{\pi u_{\alpha \beta}}{2 m} \bar{x}_{m}^{\alpha} x_{m}^{\beta} \\
& +\frac{i}{2} V \sum_{m>0}\left\{\bar{x}_{m}^{0}\left(\tilde{a}_{m}^{i_{0} \dagger}-a_{m}^{i_{0}}\right)+\left(a_{m}^{i_{0} \dagger}-\tilde{a}_{m}^{i_{0}}\right) x_{m}^{0}\right\}, \\
S_{\text {boundary }}^{f}=S_{F, T, V}^{f}=\quad & i F_{\alpha \beta} \bar{\theta}_{0}^{\alpha} \theta_{0}^{\beta}+i \sum_{r>0} F_{\alpha \beta} \bar{\theta}_{r}^{\alpha} \theta_{r}^{\beta}+i \sum_{r>0} \frac{\pi u_{\alpha \beta}}{2 r} \bar{\theta}_{r}^{\alpha} \theta_{r}^{\beta} \\
& -\frac{i}{2} V \sum_{r>0}\left\{\bar{\theta}_{r}^{0}\left(\tilde{\psi}_{r}^{i_{0}}+i \eta \psi_{r}^{i_{0} \dagger}\right)-\left(\tilde{\psi}_{r}^{i_{0} \dagger}-i \eta \psi_{r}^{i_{0}}\right) \theta_{r}^{0}\right\} .
\end{aligned}
$$


The third term in (29) is the contribution of the bulk to the boundary. We have supposed that the $D p$-brane moves with the velocity $V$ along the $X^{i_{0}}$ direction and the other components of $V^{i}$ are zero, so the presence of $V$ and the index $i_{0}$ in (29) and (30).

Boundary actions (29) and (30) help us to calculate the bosonic and fermionic boundary states according to (27) and (28):

$$
\begin{aligned}
\left|B ; S_{F, T, V}^{b}\right\rangle^{\text {bosonic }}=\quad & \prod_{m=1}\left[\operatorname{det}\left(-2 R_{(m)}\right)\right]^{-1} \exp \left(-\sum_{m=1}^{\infty} a_{m}^{\dagger} \cdot S_{(m)} \cdot \tilde{a}_{m}^{\dagger}\right)|v a c\rangle \\
& \times \frac{T_{p} \sqrt{2^{p+1}}}{\sqrt{\operatorname{det} u}} \int d p^{\alpha} \exp \left(-\frac{1}{2} P^{T} u^{-1} P\right) \delta\left(x_{0}^{i_{0}}-V x_{0}^{0}-y^{i_{0}}\right) \\
& \times \prod_{i^{\prime} \neq i_{0}} \delta\left(x_{0}^{i^{\prime}}-y^{i^{\prime}}\right) \prod_{\alpha}\left|p^{\alpha}\right\rangle \prod_{i^{\prime} \neq i_{0}}\left|p^{i^{\prime}}=0\right\rangle\left|p^{i_{0}}=V p^{0}\right\rangle, \\
\left|B ; S_{F, T, V}^{f}\right\rangle^{\text {fermionic }}=\quad & \prod_{r>0}\left[\operatorname{det}\left(-2 R_{(r)}\right)\right] \exp \left(i \eta \sum_{r>0}^{\infty} \psi_{r}^{\dagger} \cdot S_{(r)} \cdot \tilde{\psi}_{r}^{\dagger}\right)\left|v a c, \theta_{0}\right\rangle .
\end{aligned}
$$

In these boundary states, matrices $R$ and $S$ are

$$
\begin{gathered}
R_{\mu \nu}=-\frac{1}{2} g_{\mu \nu}-F_{\alpha \beta} \delta_{\mu}^{\alpha} \delta_{\nu}^{\beta}-\frac{\pi u_{\alpha \beta}}{2 r} \delta_{\mu}^{\alpha} \delta_{\nu}^{\beta}, \\
S_{\mu \nu(r)}=\frac{V^{2}}{4}\left(R_{(r)}^{-1}\right)_{00} \delta_{\mu}^{i_{0}} \delta_{\nu}^{i_{0}}+\left(R_{(r)}^{-1}\right)_{\alpha \beta} \delta_{\mu}^{\alpha} \delta_{\nu}^{\beta}+g_{\mu \nu} .
\end{gathered}
$$

where $r=m$ in the bosonic case and the integer or half-integer in the R or NS sectors of the fermionic case, respectively. The vector $P$ in the bosonic boundary state $(31)$ is defined in terms of the velocity of the $D p$-brane and the momenta of closed superstring as

$$
P_{\alpha}=V p^{i_{0}} \delta_{\alpha}^{0}-\frac{1}{2} p_{\alpha}
$$

The bosonic boundary state, (31), consists of two oscillating and zero mode parts. The first line in (31) with the infinite determinant and the exponential factor is the contribution of the oscillators which act on the $|v a c\rangle$ of oscillators. The remaining part of (31) belongs to the zero modes with some constant factors, two delta functions which have been included to determine the position of the $D p$-brane and a momentum dependent exponential which comes from taking the zero mode action into account. By integration over the momenta we consider the effect of all momentum components along the $X^{\alpha}$ directions since $P^{\alpha}$ are parallel to these directions.

Eq. (32) indicates the fermionic boundary state. Notice that the effect of the zero mode action $S_{F}^{0}=i F_{\alpha \beta} \bar{\theta}_{0}^{\alpha} \theta_{0}^{\beta}$ on the boundary state appears as a modification of the fermionic vacuum from $|v a c\rangle$ to $\left|v a c, \theta_{0}\right\rangle$. Since our goal in this section is calculating the disk partition function, which is obtained by projecting vacuum onto bra-vacuum, the only state which 
survives is $\left|v a c, \theta_{0}=0\right\rangle$. Therefore, we do not study the explicit form of $\left|v a c, \theta_{0}\right\rangle$. In fact, when $S_{F}^{0}$ acts on the fermionic vacuum, the polynomials of the $\Gamma$ matrices appear which affect the spin structure of the boundary state and is discussed in different references [3].

When all the background fields and the velocity are set to zero, $\left(R^{-1}\right)_{\alpha \beta}=-2 g_{\alpha \beta}$ and hence $S_{\mu \nu}$ decomposes into two parts, $S_{\alpha \beta}=-g_{\alpha \beta}$ and $S_{i j}=g_{i j}$. Then, this boundary state belongs to a stationary $D p$-brane without any background fields and shows that the directions $X^{\alpha}, \alpha=\{0,1, \ldots, p\}$, and $X^{i}, \alpha=\{p+1, \ldots, d-1\}$, obey Neumann and Dirichlet boundary conditions, respectively [9].

Since in the closed string theory, the disk partition function represents propagation of a closed string from the boundary of the disk and then its disappearance, so there should be a profound relation between the boundary state and disk the partition function. This relationship can be expressed as [35]

$$
\mathcal{Z}_{S_{\text {boundary }}}=\left\langle v a c \mid B ; S_{\text {boundary }}\right\rangle .
$$

The index $S_{\text {boundary }}$ indicates that the partition function is corresponding to the boundary action.

Therefore, by being equipped with the generalized bosonic and fermionic boundary states from (31) and (32), the corresponding disk partition functions are attainable according to (35). The bosonic disk partition function is

$$
\mathcal{Z}_{\text {disk }}^{b}=\frac{T_{p} \sqrt{2^{p+1}}}{\sqrt{\operatorname{det} u}} \prod_{m>0}\left[\operatorname{det}\left(-2 R_{(m)}\right)\right]^{-1} \int d p^{\alpha} \exp \left(-\frac{1}{2} P^{T} u^{-1} P\right) .
$$

The partition function (36) has the factors $1 / \sqrt{\operatorname{det} u}$ and the infinite determinant in common with conventional partition functions [15]. A significant difference is the presence of the exponential factor of momenta, which is due to inclusion of the zero mode parts of the boundary action [first three terms in (29)]. By performing the integration, the bosonic partition function takes the form

$$
\mathcal{Z}_{\text {disk }}^{b}=\frac{T_{p} \sqrt{2^{p+1}} \sqrt{(2 \pi)^{p+1}}}{2\left(V^{2}-1 / 2\right)} \prod_{m>0}\left[\operatorname{det}\left(-2 R_{(m)}\right)\right]^{-1} .
$$

After the integration over the momenta it is seen that the velocity has appeared as a coefficient and the factor $1 / \sqrt{\operatorname{det} u}$ has disappeared contrary to the case of a stationary $D p$ brane [15]. Actually, in the absence of velocity, just the tachyon contributes to the zero mode boundary action and affects the partition function by the factor $1 / \sqrt{\operatorname{det} u}$. But with the presence of velocity in the zero mode boundary action, the factor $\sqrt{\operatorname{det} u}$ appears in the partition function which cancels the former. 
Also, in the same manner fermionic partition function is derived as

$$
\mathcal{Z}_{\text {disk }}^{f}=\prod_{r>0}\left[\operatorname{det}\left(-2 R_{(r)}\right)\right],
$$

in which $r$ is the integer in the RR sector and the half-integer in the NSNS sector. As is obvious, these bosonic and fermionic partition functions actually are the coefficients of the boundary states (31) and (32).

\section{Tachyon condensation}

As previously mentioned, the presence of open string tachyon can be interpreted as $D$-brane instability and shows that we have not chosen a proper vacuum for perturbative expansion. In the other language, since our nonlinear sigma model has broken the conformal invariance the renormalization group ( $R G$ ) flow starts from a conformal fixed point and leaves for another fixed point. This RG flow occurs under the influence of tachyon, which is a relevant operator.

The tachyon potential has a minimum for $T(X)$ tending to infinity [24] that for a profile of the form $T^{2}(X)=u_{\mu \nu} X^{\mu} X^{\nu}$ is equivalent to $u \rightarrow \infty$. The transition from the UV fixed point that corresponds to the presence of an unstable $D$-brane to the IR fixed point by tachyon condensation is accompanied by the decay of an unstable $D$-brane to a stable vacuum or a stable $D$-brane. In the conventional literature, the linear evolution of a single parameter $u$ is responsible for this RG flow. Here, we have instead a multiparameter situation that is implied by the $u_{\mu \nu}$ matrix. Since we work in a flat spacetime, by writing down the beta functions it will be clear that the condensation process is independent in each coordinate. This means condensation in one direction never stimulates condensation in the other directions. It is different in curved backgrounds.

Endowed with the explicit form of the boundary states and partition functions, it seems reasonable to study the effect of tachyon condensation on them. Apart from the normalization factors of the boundary states (31) and (32), which are partition functions, the dependence of the boundary states on the parameters $u$ and $F$ is summarized in the matrix $S$, (34). It is immediately clear what happens to this matrix in the limit of infinite $u$. It will result in $S_{(r)} \rightarrow-g$.

In order to understand this result better, suppose $F=V=0$. In this artificial situation consider a $D p$-brane with a single dimensional tachyon field, $u_{p p}$, along the $X^{p}$ direction switched on, so

$$
R_{(r) \alpha \beta}=-\frac{1}{2} g_{\alpha \beta}-\frac{u_{p p}}{2 r} \delta_{\alpha}^{p} \delta_{\beta}^{p},
$$




$$
S_{(r) \mu \nu}=\left(R_{(r)}^{-1}\right)_{\alpha \beta} \delta_{\mu}^{\alpha} \delta_{\nu}^{\beta}+g_{\mu \nu}
$$

and

$$
S \rightarrow g_{i j} \oplus-g_{\alpha^{\prime} \beta^{\prime}} \oplus\left(\left(-\frac{g}{2}-\frac{u}{2 r}\right)^{-1}+g\right)_{p p} .
$$

We have decomposed the $\mu$ and $\nu$ indices into three parts: $i$ and $j$ show the perpendicular directions to the $D p$-brane, $\alpha^{\prime}$ and $\beta^{\prime}$ are used for directions parallel to the $D p$-brane world volume except $X^{p}$ and the index $p$ shows the $X^{p}$ direction. Therefore, the matrix $S$ with and without the influence of the tachyon will be

$$
S \rightarrow \begin{cases}g_{i j} \oplus-g_{\alpha^{\prime} \beta^{\prime}} \oplus-g_{p p} & u \rightarrow 0 \\ g_{i j} \oplus-g_{\alpha^{\prime} \beta^{\prime}} \oplus+g_{p p} & u \rightarrow \infty .\end{cases}
$$

Thus, with the change of the sign of $g_{p p}$, obviously, the Neumann boundary condition has been changed into a Dirichlet boundary condition. The newly generated object must therefore be a $D(p-1)$-brane. Now if in a more general situation we consider the tachyon field to have components along all the directions of the $D p$-brane world volume (i.e. $u_{\alpha \beta}$ where $\alpha, \beta=\{0,1, \ldots, p\})$, in the limit $u \rightarrow \infty$ all the $p+1$ Neumann boundary conditions convert to Dirichlet boundary conditions

$$
S \rightarrow \begin{cases}g_{i j} \oplus-g_{\alpha \beta} & u \rightarrow 0 \\ g_{i j} \oplus g_{\alpha \beta} & u \rightarrow \infty .\end{cases}
$$

This means that our $D p$-brane has lost its world volume and has reduced to a $D$-instanton. Since, the matrix $S$ has the same form in bosonic and fermionic boundary states and the investigations show the same results for integer and half-integer $r$, these arguments are valid for both the bosonic and fermionic parts.

In the next step in order to complete the tachyon condensation discussion we focus on the influence of the tachyon field on the partition functions. As mentioned before, our tachyon background generally has components along all the directions of the $D p$-brane world volume. In other words, for a $D p$-brane, $u$ is a $(p+1) \times(p+1)$ matrix, as $F$ is. Without loss of generality consider $u$ as a diagonal matrix. Here, we first study the effect of tachyon condensation on the bosonic partition function then the method would be applied to the fermionic partition functions in the RR and NSNS sectors, separately.

The only factor in (37) that includes the tachyon is $\left[\operatorname{det}\left(-2 R_{(m)}\right)\right]^{-1}$. In the first step suppose that the component $u_{p p}$ tends to infinity

$$
\begin{gathered}
\lim _{u_{p p} \rightarrow \infty} \prod_{m>1} \operatorname{det}\left(g_{\mu \nu}+\left(2 F_{\alpha \beta}+\frac{u_{\alpha \beta}}{m}\right) \delta_{\mu}^{\alpha} \delta_{\nu}^{\beta}\right)_{(p+1) \times(p+1)}^{-1} \\
=\lim _{u_{p p} \rightarrow \infty} \prod_{m>1} \operatorname{det}\left(g_{\mu^{\prime} \nu^{\prime}}+\left(2 F_{\alpha^{\prime} \beta^{\prime}}+\frac{u_{\alpha^{\prime} \beta^{\prime}}}{m}\right) \delta_{\mu^{\prime}}^{\alpha^{\prime}} \delta_{\nu^{\prime}}^{\beta^{\prime}}\right)_{p \times p}^{-1}\left(\frac{u_{p p}}{m}\right)^{-1}
\end{gathered}
$$


where

$$
\mu^{\prime}, \nu^{\prime} \in\{0,1, \ldots, d\}-\{p\} \quad \text { and } \quad \alpha^{\prime}, \beta^{\prime} \in\{0,1, \ldots, p-1\} .
$$

This means that when $u_{p p} \rightarrow \infty$, $\left[\operatorname{det}\left(-2 R_{(m)}\right)\right]^{-1}$ is changed to another determinant which has lost its $p$ dimension and a factor of $\left(u_{p p} / m\right)^{-1}$ has appeared which is equal to $\sqrt{u_{p p}}$ after regularization. So $u_{\alpha^{\prime} \beta^{\prime}}$ and $F_{\alpha^{\prime} \beta^{\prime}}$ are symmetric and antisymmetric $p \times p$ matrices, respectively. Applying the limit $u_{p p} \rightarrow \infty$ after regularization results in an infinite answer, which will be discussed later. As we continue this procedure, each time by sending any $u_{\alpha \beta}$ component to infinity, the corresponding dimension of the determinant is reduced and a factor of $\left(u_{\alpha \beta} / m\right)^{-1}$ presents. Finally, after a successive process, when all the components of $u$ are tending to infinity, the following relation is obtained

$$
\begin{gathered}
\lim _{u \rightarrow \infty} \prod_{m>1} \operatorname{det}\left(g_{\mu \nu}+\left(2 F_{\alpha \beta}+\frac{u_{\alpha \beta}}{m}\right) \delta_{\mu}^{\alpha} \delta_{\nu}^{\beta}\right)_{(p+1) \times(p+1)}^{-1}=\lim _{u \rightarrow \infty} \prod_{m>1}\left(\operatorname{det} g^{\prime}\right)^{-1}\left(\operatorname{det} \frac{u}{m}\right)^{-1} \\
=\lim _{u \rightarrow \infty} \sqrt{\operatorname{det} g^{\prime}} \sqrt{(2 \pi)^{p+1} \operatorname{det} u}
\end{gathered}
$$

where the last equality is obtained after zeta function regularization and by $g^{\prime}$ we mean the matrix of $g_{i j}$ 's.

\section{NSNS sector}

To complete the tachyon condensation process we have to study the fermionic partition function, too. The limit $u \rightarrow \infty$ of the fermionic partition function, (38), in the NSNS sector that $r$ is the half-integer, leads to the following relation:

$$
\begin{aligned}
& \lim _{u \rightarrow \infty} \prod_{r=1 / 2} \operatorname{det}\left(g_{\mu \nu}+\left(2 F_{\alpha \beta}+\frac{u_{\alpha \beta}}{r}\right) \delta_{\mu}^{\alpha} \delta_{\nu}^{\beta}\right)_{(p+1) \times(p+1)} \\
& =\lim _{u \rightarrow \infty} \prod_{r=1 / 2}\left(\operatorname{det} g^{\prime}\right) \prod_{r=1 / 2}\left(\operatorname{det} \frac{u}{r}\right)=\lim _{u \rightarrow \infty} \sqrt{\operatorname{det} u},
\end{aligned}
$$

where again the zeta function regularization has performed for the last equality. Here, because $r$ is the half-integer, $\prod_{r=1 / 2}\left(\operatorname{det} g^{\prime}\right)=1$. Therefore, the behavior of the total disk partition function when $u \rightarrow \infty$ in the NSNS sector as a combination of the bosonic, (37) and (40), and fermionic, (41), parts is given by

$$
\mathcal{Z}_{\mathrm{disk}}^{\mathrm{NSNS}}=\lim _{u \rightarrow \infty} \frac{T_{p}(2 \pi)^{p+1} \sqrt{2^{p+1}}}{2\left(V^{2}-1 / 2\right)} \sqrt{\operatorname{det} g^{\prime}} \operatorname{det} u .
$$

The relation between a $D p$-brane and a $D q$-brane tensions is $T_{p-q}=\left(2 \pi \sqrt{\alpha^{\prime}}\right)^{q} T_{p}$. This is correct for $D$-branes in bosonic string theory and also BPS branes in superstring theories. But tensions of non-BPS branes are larger by a factor $\sqrt{2}$ with respect to BPS branes [24]. So the relation between tensions of a BPS $D p$-brane and a non-BPS $D(p-1)$-brane is 
$\tilde{T}_{p-1}=2 \pi \sqrt{2 \alpha^{\prime}} T_{p}$, where $\tilde{T}$ shows the tension of a non-BPS brane. According to this relation and the point that in this article $\alpha^{\prime}=1$, we can read the tension of a non-BPS D0-brane from (42) as

$$
\tilde{T}_{0}=(\sqrt{2})^{p}(2 \pi)^{p} T_{p}
$$

There is an interpretation for this result according to the proposed system in this article. We can say that at the beginning there is a non-BPS $D p$-brane which is unstable in all $p+1$ dimensions of its world volume due to the extension of $u$ in all of these $p+1$ directions. In the limit $u \rightarrow \infty$, condensation of the $D p$-brane starts in all directions of its world volume. Depending on which components of $u$ tend to infinity, the corresponding dimensions of the $D p$-brane decrease. The resulting lower dimensional $D$-brane is still non-BPS and unstable because some components of $u$ are still available in the remaining dimensions. When the $p$ components of $u$ tend to infinity, the $D p$-brane loses its $p$ spatial dimensions and a non-BPS $D 0$-brane remains. By considering the limit $u_{p+1, p+1} \rightarrow \infty$ for the last component of $u$, the non-BPS D0-brane changes to a BPS $D$-instanton with tension

$$
T_{-1}=\frac{\tilde{T}_{0} 2 \pi}{\sqrt{2}}
$$

which is understandable from (42). In fact, the partition function (42) can be written in the following form:

$$
\mathcal{Z}_{\text {disk }}^{\mathrm{NSNS}}=\lim _{u \rightarrow \infty} \frac{T_{-1}}{\left(V^{2}-1 / 2\right)} \sqrt{\operatorname{det} g^{\prime}} \operatorname{det} u \equiv \mathcal{T}_{-1} .
$$

The factor det $u$ in the partition function (43), which is absent in conventional partition functions, stems from considering the contribution of zero modes in boundary interactions. As an explanation, det $u$ can be included in $D$-instanton tension and defines an effective tension for it, $\mathcal{T}_{-1}$. So the limit $u \rightarrow \infty$ can be translated into infinite tension. In other words, after tachyon condensation the resulting $D$-instanton has an infinite tension that is equivalent to say that even large interactions have no influence on the brane [36]. It means that no higher vibration modes are excited and one expects the brane to appear concentrated, or collapsed, in its own center of mass [37].

\section{RR sector}

In the RR sector that $r$ runs over integers, $\prod_{r=1}\left[\operatorname{det}\left(-2 R_{(r)}\right)\right]$ in the fermionic partition function cancels $\prod_{m=1}\left[\operatorname{det}\left(-2 R_{(m)}\right)\right]^{-1}$ in the bosonic one. So the total disk partition function in this sector is just constructed by the zero mode part, (37), as follows:

$$
\mathcal{Z}_{\text {disk }}^{\mathrm{RR}}=\frac{T_{p} \sqrt{2^{p+1}} \sqrt{(2 \pi)^{p+1}}}{2\left(V^{2}-1 / 2\right)} .
$$


It is seen that background fields, tachyon and gauge fields, have no contribution in this partition function. So, tachyon condensation does not change the form of the disk partition function in the RR sector. In other words, only the NSNS sector states are involved in the phenomenon of tachyon condensation.

So we studied the behavior of a kind of generalized boundary state under tachyon the condensation process. The process resembles the conventional tachyon condensation process in decreasing the dimension of the $D p$-brane. But because of simultaneous consideration of all contributions from zero modes in boundary interactions, a tachyon dependent factor remains and defines an effective tension for the newly generated $D$-brane.

\section{Summary and conclusion}

In this article we considered a moving and arbitrary dimensional $D$-brane whose background fields such as open string tachyon and $U(1)$ gauge field live on its world volume. Then we tried to couple these nonvanishing surface terms (background fields and also $D p$-brane velocity) to the main action of the theory as longitudinal and transverse boundary actions. The definition of the boundary state in terms of boundary actions helped us to calculate the boundary states with the path integral approach.

We divided these boundary states into zero and oscillating modes boundary states so that each part is constructed by the corresponding action. Inclusion of $D$-brane velocity in the problem which is absent in conventional tachyon literature, caused our zero mode boundary state to be different. Since we have taken into account the zero modes of all boundary deformations as well as the contribution of the bulk to the boundary action, the dependence of the partition function on the tachyon differs from conventional partition functions. In this case, during the tachyon condensation process the phenomena of dimensional reduction of the $D$-brane and the established relations between $D$-branes tensions occur as expected. But a tachyon dependent factor remains in the new partition function and defines an effective tension for the new lower dimensional $D$-brane.

Since we have allowed the tachyon to have components along all the directions of the $D p$-brane world volume, condensation of all $p+1$ components of tachyon field (i.e. $u$ ) results in a $D$-instanton with an effective tension that tends to infinity. This infinite tension can be interpreted as resistance against disturbances and fluctuations. Also it is verified that tachyon condensation is just definable in the NSNS sector.

Acknowledgment I would like to thank M. Baumgartl for useful discussions. Also, I am grateful to D. Kamani for his support. 


\section{References}

[1] T. Uesugi, Worldsheet Description of Tachyon Condensation in Open String Theory, hep-th/0302125.

[2] A. Sen, Int. J. Mod. Phys. A20 (2005) 5513-5656.

[3] C.G. Callan, C. Lovelace, C.R. Nappi and S.A. Yost, Nucl. Phys. B308 (1988) 221.

[4] E. Cremmer and J. Scherk, Nucl. Phys. B50 (1972) 222.

[5] C.G. Callan and I.R. Klebanov, Nucl. Phys. B465 (1996) 473.

[6] C.G. Callan, C. Lovelace, C.R. Nappi and S.A. Yost, Nucl. Phys. B288 (1987) 525; Nucl. Phys. B293 (1987) 83.

[7] M. Billo, P. Di Vecchia and D. Cangemi, Phys. Lett. B400 (1997) 63.

[8] G. Lifstchytz, Phys. Lett. B388 (1996) 720.

[9] P. Di Vecchia and A. Liccardo, NATO Adv. Study Inst. Ser. C. Math. Phys. Sci. 556 (2000) 1-59

[10] M.M. Sheikh-Jabbari, Phys. Lett. B425 (1998) 48.

[11] Z. Rezaei and D. Kamani, J. Exp. Theor. Phys. 114: 234-242, (2012), arXiv:1107.1183.

[12] T. Kitao, N. Ohta and J. Ge Zho, Phys. Lett. B428 (1998) 68.

[13] D. Kamani, Mod. Phys. Lett. A15 (2000) 1655-1664.

[14] H. Arfaei and D. Kamani, Phys. Lett. B452 (1999) 54; Nucl. Phys.B561 (1999) 57 .

[15] T. Lee, Phys. Rev. D64 (2001) 106004.

[16] A. Sen, JHEP 0405 (2004) 076.

[17] E. T. Akhmedov, M. Laidlaw and G. W. Semenoff, JETP Lett. 77: 1-6,2003, PismaZh. Eksp. Teor. Fiz. 77: 3-8, 2003. 
[18] D. Kutasov, M. Marino and G. Moore, JHEP 0010 (2000) 045.

[19] T. Lee, Phys. Lett. B 520 (2001) 385390.

[20] K. Hashimoto, P. M. Ho and J. E. Wang, Mod. Phys. Lett. A20 (2005)79-94.

[21] A. Lerda and R. Russo, Int. J. Mod. Phys. A15 (2000) 771.

[22] A. Sen, Int. J. Mod. Phys. A14 (1999) 4061-4078.

[23] M. Hindmarsh and H. Li, JHEP 0906 (2009) 050.

[24] D. Kutasov, M. Marino and G. Moore, Remarks on Tachyon Condensation in Superstring Field Theory, hep-th/0010108.

[25] V.A. Kostelecky, S. Samuel, Phys. Lett. B 207 (1988) 169; Nucl.Phys. B 336 (1990) 263.

[26] A. Sen, B. Zwiebach, JHEP 0003 (2000) 002.

[27] N. Moeller, W. Taylor, Nucl. Phys. B 583 (2000) 105.

[28] E. Witten, Phys. Rev. D 46 (1992) 5467; Phys. Rev. D 47 (1993) 3405.

[29] S.L. Shatashvili, Phys. Lett. B 311 (1993) 83.

[30] A.A. Gerasimov, S.L. Shatashvili, JHEP 10 (2000) 034.

[31] P. Kraus, F. Larsen, Phys. Rev. D 63 (2001) 106004.

[32] T. Takayanagi, S. Terashima, T. Uesugi, JHEP 0103 (2001) 019.

[33] M. Baumgartl, On the Correspondence of Open and Closed Strings, 0812.2361.

[34] E. Bergshoeff, E. Sezgin, C.N. Pope and P.K. Townsend, Phys. Lett. B 188 (1987).

[35] G. Arutyunov, A. Pankiewicz and B. Stefanski Jr., JHEP 06 (2001) 049.

[36] A. Zimmerman Jones, D. Robbins, String Theory For Dummies, Wiley Publishing Inc., 2010.

[37] S. Ansoldi, A. Aurilia, C. Castro, E. Spallucci, Phys. Rev. D64 (2001) 026003. 